Conclusion Acceptability and feasibility of STI screening among pregnant women in Vietnam was very high. The prevalence of CT was much higher than the mean prevalence of CT among low and middle income countries in Asia. Study findings support policy to incorporate routine screening for STIs during pregnancy to improve maternal and infant health.

\section{P3.160 OPTIMISING EXISTING SEXUAL HEALTH CLINICS INCREASES HIV TESTING AMONG GAY AND BISEXUAL MEN AT HIGHER RISK OF INFECTION}

${ }^{1}$ Muhammad Shahid Jamil, ${ }^{1}$ Hamish Mcmanus, 'Denton Callander, 'Garrett Prestage, ${ }^{1}$ Hammad Ali, ${ }^{2}$ Vickie Knight, ${ }^{3}$ Tim Duck, ${ }^{4}$ Catherine C O'connor, ${ }^{5}$ Marcus Chen, ${ }^{2}$ Anna M Mcnulty, ${ }^{1}$ Phillip Keen, ${ }^{5}$ Nick Medland, ${ }^{6}$ Margaret Hellard, ${ }^{7}$ Andrew E Grulich, ${ }^{8}$ David A Lewis, ${ }^{1}$ John M Kaldor, ${ }^{5}$ Christopher K Fairley, 'Basil Donovan, ${ }^{1}$ Rebecca J Guy. ${ }^{1}$ The Kirby Institute, Unsw Australia, Sydney, Australia; ${ }^{2}$ Sydney Sexual Health Centre, Sydney Hospital, Sydney, Australia; ${ }^{3}$ Nsw Ministry of Health, Sydney, Australia; ${ }^{4}$ Sexual Health Service, Community Health, Sydney Local Health District, Sydney, Australia; ${ }^{5}$ Melbourne Sexual Health Centre, Melbourne, Australia; ${ }^{6}$ Centre for Population Health, Burnet Institute, Melbourne, Australia; 7 The Kirby Institute, Unsw Australia, Sydney, Australia, Sydney, Australia; ${ }^{8}$ Western Sydney Sexual Health Centre, Sydney, Australia

\subsection{6/sextrans-2017-053264.395}

Introduction Globally, community-based HIV testing models are recommended to improve access to testing with less focus on optimising existing clinical services. In the past 5 years, public-funded sexual health clinics (SHCs) in New South Wales (NSW, Australia) have taken a range of initiatives to improve efficiencies such as triage to divert low-risk heterosexuals, express clinics, online booking, self-registration and SMS reminders. We analysed temporal trends in HIV testing among gay and bisexual men (GBM) attending SHCs in this period and assessed if testing was targeted to high-risk GBM.

Methods We used retrospective data from 32 SHCs in NSW participating in a surveillance network. HIV-negative GBM were categorised based on client type (new or existing), risk status (using partner numbers and/or recent rectal sexually transmitted infection), and recent HIV testing (past 6 months for high-risk, past 12 months for low-risk GBM). We used repeated measures Poisson regression to assess trends in attendance, tests and contribution to total tests by GBM categories.

Results From 2009-2015, unique GBM attending increased by $82 \%(5,477$ to 9,983$)$, and HIV tests increased by $155 \%$ $(4,779$ to 12,173$)$ with significant increase in all categories and greatest increase in existing high-risk clients. Of 58,377 HIV tests done, $74 \%$ were in existing and 35\% in high-risk clients. Over time, existing high-risk clients with recent testing had an increasingly larger contribution to total tests $(13 \%$ annual increase, 95\% CI:8\%-18\%,p<0.001). There was a simultaneous annual decline in contribution by these low-risk categories: new clients $(5 \%$ decline, $95 \% \mathrm{CI}: 2 \%-7 \%$, $\mathrm{p}<0.001)$; existing clients with no recent testing $(6 \%$ decline, $95 \% \mathrm{CI}: 5 \%-7 \%, \mathrm{p}<0.001)$. There were no changes in contribution by other categories (new high-risk clients; existing high-risk clients with no recent testing; existing low-risk clients with recent testing).

Conclusion SHCs in NSW have successfully increased HIV testing among GBM, with greatest increase in high-risk men. The strategies adopted could be translated to other settings.
P3.161 FACTORS ASSOCIATED WITH POSITIVE TESTS FOR HIV E SYPHILIS CENTRE AND ADVICE

${ }^{1}$ Nara Borges Gonçalves Lima, ${ }^{1}$ Heber José De Moura, ${ }^{2}$ Roumayne Fernandes Vieira Andrade, ${ }^{1} E$ Elk De Assis Araújo, ${ }^{1}$ Maria Alix Leite Araújo. 'Universidade de Fortaleza, Fortaleza - CE, Brazil; ${ }^{2}$ Faculdade de Ciências Médicas, Campina Grande - PB, Brazil

\subsection{6/sextrans-2017-053264.396}

Introducion Faced with rapid evolution of sexually transmitted infections (STIs), especially HIV/AIDS, as well as the impacts on society, this study aimed to analyse the factors associated with seropositivity TR for HIV and syphilis in people served in the Testing Centre and counselling - CTA Fortaleza, Ceará. Methods A cross-sectional study that analysed data from Forms Customer Information System of people who performed the rapid test (RT) in the months of June and July 2015. We analysed sociodemographic, behavioural and epidemiological variables. The data were entered into the Statistical Package for the Social Sciences and were analysed using frequency distributions, measures of central tendency and dispersion.

Results Were analysed 882 forms. The research result shows that $622(70.5 \%)$ were males and 414 (46.9\%) were aged between 19 and 29 years, with a predominance of non-white people, without a partner, with more than four years of study, coming from Fortaleza. Of the 875 tests for HIV and 648 tests for syphilis destecção, 49 (5.3\%) and 72 (8.1\%) showed reagent test result, respectively. Cases of HIV reagent were associated with the identification of the service by friend/service user $(p=0.013)$, male gender $(p=0.006)$, non-Caucasians $(p=0.045)$, lower education of four years of study $(p=0.045)$ and working $(p=0.009)$. There was statistical association in positive cases of syphilis and present IST $(\mathrm{p}<0.001)$ and hoemens sexual partners and women $(p=0.012)$.

Conclusion These findings suggest the need for effective measures for control and prevention of STIs aimed at young adults, male and more than one sexual partner.

\section{P3.162 DEVELOPING A CLINICAL PREDICTION RULE TO TARGET STI TESTING AND CONTRACEPTION TO WOMEN IN COMMUNITY SETTINGS: IMPLICATIONS FOR SEXUAL HEALTH SURVEYS}

${ }^{1}$ Natalie Edelman, ${ }^{2}$ De Visser Ro, ${ }^{3}$ Mercer Cm, ${ }^{1} \mathrm{~J}$ Cassell. 'Brighton and Sussex Medical School, Brighton, UK; ${ }^{2}$ University of Sussex, Brighton, UK; ${ }^{3}$ University College London, London, UK

\subsection{6/sextrans-2017-053264.397}

Introduction Applied sexual health surveys investigate associations between psychosocial and demographic factors, sexual risk behaviour, uptake of interventions and sexual morbidity. This work improves clinical and public health understandings of sexual morbidity but may not be easily translated into practice. We seek to identify key features of research surveys that would make them more relevant to the development and use of risk assessment and targeting tools.

Methods To support the development of clinically-embedded risk assessment tools we undertook a systematic review of population surveys reporting on sexual risk and morbidity. We identified aspects of sexual health research questionnaire items and their reporting which could be adapted to better serve public health and clinical services to identify patients for targeted intervention. 